\title{
ИНФОРМАЦИОННАЯ ОБРАЗОВАТЕЛЬНАЯ СРЕДА БАЙКАЛЬСКОГО ГОСУДАРСТВЕННОГО УНИВЕРСИТЕТА
}

\begin{abstract}
АНнотАЦИя. В статье рассматриваются требования, предъявляемые ФГОС ВО к информационной образовательной среде вуза. Отмечается частичное выполнение этих требований в Байкальском государственном университете. Предложена технология формирования электронного портфолио студента, включающая централизованное хранение данных в базе данных, загрузку данных студентами по локальной сети и сети Интернет, доступ преподавателей к данным студентов, передачу работ студентов в библиотечную систему, строгое разграничение полномочий всех пользователей, имеющих доступ к портфолио студентов. Обосновывается актуальность автоматизированного создания основных профессиональных образовательных программ. Разработана информационная модель рабочей программы дисциплины. Описаны технология применения разработанных средств автоматизации, процедуры контроля, применение электронных рабочих дисциплин в электронной образовательной среде, формирование паспортов компетенций, анализ обеспеченности дисциплин, контроль процесса формирования компетенций на основе предложенной модели рабочей программы дисциплины и мобильного приложения для ввода данных посещаемости и текущей успеваемости.

КЛЮЧЕВЫЕ СЛОВА. Информационная образовательная среда; портфолио студентов; ФГОС ВО; разграничение доступа; портал студента; АРМ преподавателя; компетенции; модель программы дисциплины; оценивание компетенций; латентные параметры. ИНФОРМАЦИЯ О СТАТЬЕ. Дата поступления 7 марта 2017 г.; дата принятия к печати 22 марта 2017 г.; дата онлайн-размещения 31 марта 2017 г.
\end{abstract}

V. V. Bratishchenko Baikal State University, Irkutsk, Russian Federation

\section{INFORMATION AND EDUCATIONAL ENVIRONMENT IN BAIKAL STATE UNIVERSITY}

ABSTRACT. The article considers the requirements set by the Federal Education Standard of Higher Education for the information and educational environment of the university. It notes a partial fulfillment of these requirements in BSU. It offers a technology of forming the student's electronic portfolio, including centralized storage of the data in the database, download of the data by students through the local network and the Internet, access of the faculty members $s$ to the students data, transfer of students' work to the library system, strict differentiation of authorities for every user that have access to the students' portfolio. The article substantiates the topicality of automated creation of basic professional educational programs. It develops an information model of the working program for the academic discipline. It describes a technology of using the developed automation means, the control procedure, application of electronic academic disciplines in the electronic educational environment, formation of competence passports, analysis of academic subject provision, control of formation process for competences on the basis of the proposed model of the working program for the discipline and mobile application for entering the data on attendance and current academic performance.

KEYWORDS. Informational educational environment; students' portfolio; Federal Education Standard of Higher Education; student's portal; WKS for faculty staffers; competences; model of discipline program; competence evaluation; latent parameters. ARTICLE INFO. Received March 7, 2017; accepted March 22, 2017; available online March 31, 2017.

(C) В. В. Братищенко, 2017

\section{Baikal Research Journal}


Современные федеральные государственные образовательные стандарты высшего образования утверждают, что «Электронная информационно-образовательная среда организации должна обеспечивать:

- доступ к учебным планам, рабочим программам дисциплин (модулей), практик, к изданиям электронных библиотечных систем и электронным образовательным ресурсам, указанным в рабочих программах;

- фиксацию хода образовательного процесса, результатов промежуточной аттестации и результатов освоения программы;

- проведение всех видов занятий, процедур оценки результатов обучения, реализация которых предусмотрена с применением электронного обучения, дистанционных образовательных технологий;

- формирование электронного портфолио обучающегося, в том числе сохранение работ обучающегося, рецензий и оценок на эти работы со стороны любых участников образовательного процесса;

- взаимодействие между участниками образовательного процесса, в том числе синхронное и (или) асинхронное взаимодействие посредством сети Интернет» ${ }^{*}$

Цель выполнения этих требований очевидна - сделать процесс учебной работы прозрачным, управляемым и контролируемым для исключения следующих достаточно распространенных негативных явлений:

1. Плагиат - списывание с просторов Интернета готовых рефератов, курсовых и выпускных квалификационных работ. Даже ответы на простые вопросы («В чем измеряется сила тока?») современный студент предпочитает находить в мировой паутине. Ситуация становится все более острой - студенты не только не получают навыка в решении задач, но и зачастую не могут отличить правильное решение от неправильного, в силу привычки подставлять первый попавшийся вариант ответа и отсутствия знаний и навыков критического осмысления найденного решения. Этому же способствуют привычки сдавать экзамены на манер единого государственного экзамена (ЕГЭ) в тестовой форме.

2. Отсутствие «прозрачности» в выставлении оценки. Как правило, оценивание остается почти интимным действием двоих: педагога и студента. Бесконтрольность этого процесса является основой для коррупционных действий преподавателей. «Выкладывание» наиболее значимых результатов учебной работы (рефератов, курсовых работ, выпускных квалификационных работ) создаст условия для проверки оценочной деятельности преподавателей. Это также позволит создать здоровую конкурентную среду среди студентов, будет стимулировать создание лучших образцов, выработку критического взгляда (который всегда лучше формировать, находя недостатки в чужих работах). Конечно, такие работы должны сопровождаться грамотным и обоснованным разбором достоинств и недостатков предлагаемых решений и формулировкой выводов со стороны преподавателя. Это большая работа, но она и измеряется значительным количеством часов работы преподавателя, выделенных на проверку и руководство студенческими работами. Отсутствие рецензии на работы студентов может превратить такую работу в халтуру, когда оценка выставляется по инерции, без должного обоснования.

3. Отсутствие контроля за сроками выполнения работ. Ведение журналов приема и выдачи работ является достаточно трудоемким, и представляется архаичным в эпоху современных информационно-коммуникационных технологий. Информационно-образовательная среда позволяет без лишних усилий регистри-

${ }^{1}$ Об утверждении федерального государственного образовательного стандарта высшего образования по направлению подготовки 09.03.03 Прикладная информатика (уровень бакалавриата) : приказ Минобрнауки России от 12 марта 2015 г. № 207 (зарегистрировано в Минюсте России 27 марта 2015 г. № 36589) // СПС «КонсультантПлюс».

\section{Baikal Research Journal}

электронный научный журнал Байкальского государственного университета 
ровать факты приемки и сдачи работ, хотя и требует от всех участников процесса определенной дисциплины, добиться которой и поможет статистика по исполнению нормативов на выполнение студентами и проверку их преподавателями.

В Байкальском государственном университете (БГУ) информационная система управления учебным процессом $[1 ; 2]$ функционирует свыше 18 лет. За этот период привычными стали электронное расписание, электронный каталог научной библиотеки университета, электронный журнал учета нагрузки преподавателя, картотека методических и научных трудов, электронное личное дело студента, организационно-методическое обеспечение ученого процесса, предоставляемое студентам по интернету в его личном кабинете. Таким образом, выполнение двух первых требований ФГОС ВО выполнялось в БГУ задолго до разработки и внедрения этих стандартов.

Дистанционные технологии в БГУ реализованы в свободно распространяемой системе дистанционного обучения Moodle [3]. В данной системе реализовано значительное количество электронных курсов. Внедрению дистанционных технологий препятствуют два обстоятельства. Во-первых, это слабая мотивировка студентов (прежде всего, очной формы обучения) самостоятельного изучения дисциплины. Во-вторых, высокая трудоемкость разработки дистанционного курса.

Таким образом, учет в информационной системе БГУ ограничивается только промежуточным, сессионным контролем, по-прежнему позволяя студенту «весело жить от сессии до сессии", и не отражает содержательных итогов учебной работы студента. Все это привело к новому этапу развития информационно-образовательной среды БГУ, который характеризуется новыми возможностями для всех его участников.

Из возможных вариантов построения информационно-образовательной среды выбрали один - предусматривающий централизованное хранение всех данных учебного процесса. Достоинствами такой архитектуры является строгая регламентация доступа всех участников учебного процесса:

- студент получает возможность хранить результаты учебной работы в виде файлов с доступом к ним только автора и проверяющих,

- преподаватель может ознакомиться и оценить результаты работы по своим предметам у своих студентов,

- декан и заведующий кафедрой получают функции контроля своевременности и качества работы и студентов, и преподавателей.

Дополнительный положительный эффект должно дать информирование родителей (спонсоров) студентов о выполнении графика учебы и результатах учебной работы. Не секрет, что отсутствие оперативного контроля за выполнением студентами учебных обязанностей является основным источником академической неуспеваемости, особенно на первых курсах.

В этой архитектуре центральное место занимает система баз данных, обеспечивающая надежное хранение данных и строго регламентированный доступ. Доступ к данным предоставляется по локальной вычислительной сети через систему приложений для всех участников учебного процесса: студента, преподавателя, заведующего кафедрой, декана, проректора по учебной работе. На основании учетных данных в базе и результатов аутентификации пользователя приложение настраивается на контекст и систему полномочий каждого. Для студента дополнительно предоставляется доступ к контенту обучения через личный кабинет на портале БГУ.

В личном кабинете студент может посмотреть сведения из личного дела, оценки, расписание занятий, учебный план, методические материалы, привязанные к дисциплинам учебного плана. В один клик он открывает материалы, подготовленные преподавателем, электронные библиотечные источники или файлы,

\section{Baikal Research Journal}

электронный научный журнал Байкальского государственного университета 
присоединенные к библиографическому описанию в научной библиотеке. В БГУ, как правило, подготовленные преподавателями учебные пособия передаются в библиотеку сразу в электронном виде, каталогизируются и сразу становятся доступными в локальной сети и через Интернет для авторизованных читателей.

С 2016 г. студент БГУ получил возможность не только размещать разработанные в рамках учебных заданий файлы, но и отправлять их на проверку преподавателям. Преподаватель, в свою очередь, в своем приложении видит список работ, присланных ему студентами. Для каждой работы он может сформировать резюме и установить статус работы. Статус «проверен» блокирует любые изменения файла.

На этой технологии реализован регламент работы над выпускными квалификационными работами (ВКР) студентов. На первом этапе в информационную систему БГУ вводятся сведения о темах и руководителях ВКР. Разработанные ВКР передаются студентами через личный кабинет руководителям. Руководитель может либо принять работу и выставить соответствующую оценку (работа получает статус «проверено»), либо сформулировать замечания и вернуть работу для исправления. После выставления оценки изменения работы блокируются и выполняется проверка ВКР на плагиат. В случае превышения критического порога заимствований с ВКР снимается блокировка для ее исправления. Полностью готовая и защищенная работа передается в научную библиотеку БГУ для размещения в открытом доступе для студентов и сотрудников. По данным АСУ контролируется выполнение графика работы над ВКР.

В целом переход на данную технологию должен позволить с наименьшими затратами сформировать электронное портфолио каждого студента, которое, кроме сессионных оценок, будет содержать объективные данные о выполнении студентом графика учебы и наиболее значимые учебные результаты. Это может и должно дать большой положительный эффект для повышения качества ВКР и всего учебного процесса в целом.

Нормативно-справочной информации, предоставляемой студентам по сети Интернет и приложение «Студент» недостаточно для организации самостоятельной работы студентов. Дополнительно требуется предоставление учебно-методических материалов по всем дисциплинам и видам учебной работы. Для выполнения этого преподаватели разрабатывают рабочие программы дисциплин (РПД), составляющие основную часть основной профессиональной образовательной программы (ОПОП). Кроме этого, необходимо обеспечить студентам доступ к РПД по профилю его подготовки. В БГУ автоматизировано создание РПД, сохранение данных в информационно-образовательной среде, и предоставление авторизованного доступа к ним и студентам, и преподавателям.

В средствах автоматизации формирования ОПОП сделана попытка разделить содержательную часть РПД (темы дисциплины, лекции, семинары, учебные задания текущей успеваемости и оценочные средства результатов их выполнения, описание промежуточных аттестаций) и организационную часть РПД (направления и профили подготовки, место дисциплины в рабочем учебном плане, часы занятий, трудоемкость в зачетных единицах).

Автоматизированная система управления (АСУ) БГУ к моменту реализации проекта уже содержала нормативные данные для описания рабочих учебных планов: направления и профили подготовки, графики изучения дисциплин, прохождения практик, промежуточных и итоговых аттестаций. В дополнении была разработана информационная модель описания рабочей программы дисциплины, включающая следующие компоненты:

- цели и задачи изучения дисциплины;

- список предшествующих дисциплин;

\section{Baikal Research Journal}

электронный научный журнал Байкальского государственного университета 
- описания промежуточных аттестаций дисциплины: разделы (виды заданий) аттестации, варианты вопросов, тестов, заданий;

- темы с указанием часов лекций, семинаров, самостоятельной работы;

- лекции (наименование, содержание) с привязкой к темам;

- семинары (наименование, содержание) с привязкой к темам;

- фонды оценочных средств дисциплины - задания (наименование, содержание) с привязкой к темам;

- библиотечные источники, электронные источники из электронных библиотечных систем, методические разработки, программное обеспечение, прочие ресурсы;

- справочные данные: ФГОСы, компетенции, виды деятельности, группы компетенций.

Наиболее сложным является описание заданий. Прежде всего, задания могут быть различных видов: задачи, тесты, case-study, доклады, деловые игры, эссе и другие. Кроме этого, каждое задание может быть связано с несколькими компетенциями, знаниями, умениями или навыками. Для исключения неопределенных многозначных связей задания разделены на компоненты. Каждый компонент может быть связан с одной компетенцией, знанием, умением, навыком. Каждый компонент может быть оценен от нуля до установленного для компонента максимального количества баллов. Для каждого компонента устанавливается критерий оценивания. Сумма баллов компонент образует оценку задания, сумма баллов заданий - оценку дисциплины по итогам текущей успеваемости.

Ввод РПД реализован в приложении «Преподаватель». Доступ к РПД строго регламентирован. Для каждого рабочего учебного плана в АСУ регистрируется ответственный за ОПОП, который вводит и корректирует матрицу компетенций, устанавливающую соответствие компетенций и дисциплин, контролирует изготовление РПД и отмечает готовность РПД, прошедших проверку. В приложении «Кафедра» заведующий кафедрой устанавливает для каждой дисциплины ответственного за разработку РПД. Каждый преподаватель в своем приложении получает список дисциплин, за разработку которых он отвечает. После ввода всех компонент автор отмечает готовность РПД.

Система контролирует набор формальных параметров РПД:

- соответствие рабочему учебному плану по часам;

- соответствие матрице компетенций;

- наличие всех необходимых компонент описания;

- сумма всех баллов текущей успеваемости, предшествующей промежуточной аттестации, должна составлять 100 баллов;

- обеспеченность литературой;

- наличие критериев выставления оценки.

Формальный контроль позволяет исключить технические ошибки при вводе описания РПД. Прошедшие формальную и содержательную проверку РПД блокируются от изменений.

В организационном плане система позволяет получать сводную и детальную информацию по каждому этапу формирования ОПОП: назначение ответственных за ОПОП и разработку РПД, ввод РПД авторами, контроль РПД ответственными за ОПОП. Эта информация предоставляется всем участникам процесса: проректору по учебной работе, начальнику учебно-методического управления, начальнику методического отдела, заведующим кафедрами, ответственным за ОПОП, преподавателям.

В случае изменений в учебных планах аннулируются результаты проверок и с соответствующих РПД снимаются блокировки изменений. Соответствующие корректировки (как правило, в объемах нагрузки в часах и кредитах) вносятся автором РПД, после чего снова выполняется контроль ответственным за ОПОП.

\section{Baikal Research Journal}

электронный научный журнал Байкальского государственного университета 
Благодаря локализации изменений авторы РПД получают точную информацию о несоответствиях и оперативно выполняют корректировки.

Внедрение ФГОС в установленные сроки (как правило, в течение 10 дней со дня опубликования) приводит к несоответствиям в рабочих учебных планах одной образовательной программы для разных лет поступления. Поэтому РПД разных студенческих потоков могут отличаться некоторыми параметрами и формируются отдельно для каждого потока. Для выполнения этой работы с минимальными затратами в АСУ разработана процедура копирования РПД. Это позволило разработанную РПД тиражировать не только в рамках одной образовательной программы, но и между разными программами и направлениями. Как правило, после такого копирования автору остается только поправить часы распределения работы по темам, хотя конечно можно выполнять другие изменения.

Централизованное хранение рабочих программ дисциплин в системе позволило решить и другие задачи в соответствии с требованиями ФГОС. Прежде всего, это касается методического обеспечения дисциплин. В РПД предусмотрена возможность включать тексты или ссылки на методические материалы. Каждый студент получает авторизованный доступ ко всем РПД программы обучения с помощью приложения «Студент», доступного в локальной вычислительной сети университета, или через личный портал в Интернете.

Включение в РПД данных об использовании библиотечных и электронных источников создает условия оптимизации учебных фондов библиотеки. Для каждого библиотечного или электронного источника собираются ссылки из РПД. Сведения о контингенте студентов позволяют точно рассчитать книгообеспеченность каждой дисциплины ОПОП. Кроме этого, можно выделить учебные фонды, не востребованные учебным процессом.

Предложенная структура описания ОПОП позволяет формировать паспорта компетенций. Для этого для каждой компетенции выбираются связанные с ней компоненты заданий из разных дисциплин, строится список соответствующих знаний, умений и навыков, а также оценочных процедур. Проблема заключается в методическом переосмыслении оценочных процедур. Предложенный подход требует более тщательного структурирования оценочных средств. До внедрения компетентностного подхода задания в текущей успеваемости редко были связаны с одной компетенцией, а в предложенной схеме это требование становится основным для корректного описания процесса изучения дисциплины в РПД.

Модель РПД позволяет собирать данные для оценивания степени сформированности компетенций по результатам текущей успеваемости. Наиболее трудоемкая процедура регистрации оценок должна выполняться немедленно и по месту оценивания. Идеальным средством для этого являются мобильные приложения, установленные на смартфонах или планшетах. Справочные данные в таких приложениях: списки обучающихся, графики работы, задания, оцениваемые компоненты (компетенции), соответствующие знания, умения и навыки - должны просто появляться на экране для обеспечения ввода оценки «в одно касание».

Разработанное в БГУ мобильное приложение предоставляет интерфейс для ввода посещаемости и текущих оценок. Сведения о студентах, организации учебного процесса и оценки текущей успеваемости целесообразно хранить в единой базе данных, связь с которой может быть обеспечена по сети WI-FI или по сети Интернет соответствующим программным обеспечением. В разработанной версии [4] мобильное приложение взаимодействует с ASP.NET приложением на базе веб-сервера Microsoft IIS для организации взаимодействия мобильного приложения с сервером базы данных и сервером Active Directory. Сервер Active Directory используется для предоставления сервисов авторизации и аутентификации поль-

\section{Baikal Research Journal}

электронный научный журнал Байкальского государственного университета 
зователей. MS SQL сервер обеспечивает хранение и авторизованный доступ ко всем данным учебного процесса.

После аутентификации мобильное приложение настраивается на контекст работы преподавателя: предлагает выбрать занятия в соответствии с его расписанием в базе данных, формирует списки соответствующих групп студентов, для выбранных группы и дисциплины демонстрирует набор заданий и компонентов из РПД. Используя мобильное приложение, преподаватель может отмечать присутствие студента или вводить баллы за выполненный компонент задания. Все действия в мобильном приложении выполняются выбором на экране. Введенные данные записываются в базу данных системы.

Разработанные средства учета текущей успеваемости позволяют регистрировать и накапливать оценки за компоненты заданий. Следующая задача - объединение этих оценок для получения итоговых оценок по дисциплине или по компетенции - может решаться несколькими методами. Итоговую оценку можно получить как средневзвешенную оценку компонент. Если в рамках одной дисциплины этот подход вполне оправдан - оценивание проводит один и тот же преподаватель, то в отношении оценки компетенций такое усреднение может привести к искажению результата, потому что разные преподаватели могут вкладывать разный смысл в одинаковые баллы, т. е. проводить измерения в разных шкалах, даже если они имеют одинаковые обозначения. Поэтому перспективным вариантом измерения сформированности компетенции является теория латентных переменных [5], успешно применяемая для обработки результатов тестирования и исследований в социально-экономических системах.

Теория латентных переменных (в англоязычном варианте Item Response Theory, IRT) вводит две основные характеристики: «подготовленность» студента и «сложность» задания. Данные характеристики влияют на вероятность правильного решения задания студентом. Вероятность правильного решения описывается функцией двух латентных параметров:

$-\delta$ - параметр, характеризующий «сложность» задания;

- $\theta$ - параметр, характеризующий «подготовленность» студента.

Латентность данных параметров означает, что их нельзя измерять непосредственно. Для оценки компетенций параметр «подготовленности» можно интерпретировать как уровень сформированности компетенции.

Простейшая модель IRT была предложена Георгом Рашем и предназначена для описания вероятности правильного ответа на дихотомическое задание, в котором возможно только две оценки: 0 или 1, т. е. решение может быть либо правильным, либо неправильным [6]. В случае с балльной системой оценивания, оценка может быть политомической - иметь множество градаций. В теории латентных переменных существуют различные модели, применимые к политомическим оценкам. Известные модели вводят латентные параметры для каждого варианта ответа - значения оценки от 0 до максимального балла. Предполагается, что все варианты ответа ранжированы по трудности.

В модели Ratings Scale Model латентные параметры одинаковых по номеру вариантов ответа для разных заданий одинаковы [7]. Это означает, что в разных тестовых заданиях «шаг трудности» между вариантами ответов одинаковый, хотя «трудности» заданий могут быть разными. Это предъявляет специфические требования к составлению таких заданий.

В модели Partial Credit Model латентные параметры одинаковых по номеру вариантов ответа для разных заданий различны [8]. Ее особенностью является большое количество параметров, для надежной оценки которых требуется значительное количество наблюдений.

\section{Baikal Research Journal}


Достаточно часто (например, в заданиях с множественным выбором или с установлением соответствия) результаты тестовых заданий получаются как сумма ответов на отдельные пункты задания. Многие тестовые системы в таких случаях предлагают более гибкие системы оценок. Не просто «правильно» - 1 и «неправильно» - 0 , а определенное количество баллов от 0 до максимального значения. Можно предположить, что распределение ответов в таких заданиях будет близким к биномиальному.

Для описания таких заданий предлагается [4; 9] применять биномиальное распределение

$$
P\left\{X_{i j}=k\right\}=C_{l_{j}}^{k} p_{i j}{ }^{k} q_{i j}^{l_{j}-k}, k \in\left\{0,1, \ldots, l_{j}\right\},
$$

где $X_{i j}-$ случайная величина ответа $i$-го тестируемого $(i=1, \ldots, n)$ на $j$-е задание $(j=1, \ldots, m)$. Параметры

$$
\begin{gathered}
p_{i j}=\frac{e^{\theta_{i}}}{e^{\theta_{i}}+e^{\delta_{j}}}, \\
q_{i j}=1-p_{i j}=\frac{e^{\delta_{j}}}{e^{\theta_{i}}+e^{\delta_{j}}},
\end{gathered}
$$

биномиального распределения, как и в модели Раша, зависят от $\theta_{i}$ - уровня подготовленности $i$-го тестируемого и $\delta_{j}-$ уровня трудности $j$-го задания.

Множество оценок позволяет получить, с одной стороны, оценки латентных параметров сформированности компетенции каждого студента, с другой - оценки латентных параметров трудности заданий. Значительное количество текущих оценок увеличивает достоверность статистических выводов. Совокупность латентных параметров точнее описывает результаты обучения чем усредненные баллы по предметам для каждого студента или усредненные баллы по студентам для каждого предмета. Латентные параметры помогут исключить из процесса обучения неоправданно легкие или сложные задания.

Предложенная система разработки и применения РПД прошла апробацию в БГУ и в настоящее время успешно применяется для создания пакета документов в соответствии с требованиями ФГОС. Разработанные на ее основе средства учета текущей успеваемости и посещаемости позволяют организовать достаточно полный контроль за процессом овладения компетенциями, накапливать детальные данные успеваемости, и за счет всестороннего анализа повысить качество обучения.

\section{Список использованной литературы}

1. Современные информационно-телекоммуникационные технологии в управлении социально-экономическими системами / А. А. Суходолов, Т. Г. Озерникова, В. В. Братищенко [и др.] ; под общ. ред. А. П. Суходолова. - Иркутск : Изд-во БГУЭП, 2013. - 196 с.

2. Братищенко В. В. Автоматизированная система управления Байкальского государственного университета экономики и права / В. В. Братищенко // Вестник Московского городского педагогического университета. Сер.: Информатики и информатизация образования. - 2007. - № 2 (9). - С. 156-158.

3. Белозубов А. В. Система дистанционного обучения Moodle : учеб.-метод. пособие / А. В. Белозубов, Д. Г. Николаев. - СПб. : С.-Петерб. нац. исслед. ун-т информ. технологий, механики и оптики (Ун-т ИТМО), 2007. - 108 с.

4. Братищенко В. В. Модель с латентными параметрами для оценивания компетенций студентов по данным текущей успеваемости / В. В. Братищенко, К. А. Кешиков // Известия Байкальского государственного университета. - 2016. - Т. 26, № 5. - С. 811-817. DOI: $10.17150 / 2500-2759.2016 .26(5) .811-817$.

5 . Нейман Ю. М. Введение в теорию моделирования и параметризации педагогических тестов / Ю. М. Нейман, В. А. Хлебников. - М. : Прометей, 2000. - 168 с.

\section{Baikal Research Journal}


6. Rasch G. Probabilistic models for some intelligence and attainment tests / G. Rasch. Chicago : The University of Chicago Press, 1980. - $199 \mathrm{p}$.

7. Andrich D. A rating formulation for ordered response categories / D. Andrich // Psychometrika. - 1978. - Vol. 43, № 4. - P. 561-573.

8. Masters G. N. A Rasch model for partial credit scoring / G. N. Masters / Psychometrika. 1982. - Vol. 47, № 2. - P. 149-174.

9. Братищенко В. В. Параметрическая модель экзаменационных оценок / В. В. Братищенко // Качество. Инновации. Образование. - 2012. - № 3, вып. 82. - С. 32-35.

\section{References}

1. Sukhodolov A. P., Ozernikova T. G., Bratishchenko V. V. et al. Sovremennye informatsionno-telekommunikatsionnye tekhnologii $v$ upravlenii sotsial'no-ekonomicheskimi sistemami [Modern Information and Telecommunication Technologies for Social and Economic Management]. Irkutsk, Baikal State University of Economics and Law Publ., 2013. 196 p.

2. Bratishchenko V. V. Automated system of management in Baikal State University of Economics and Law. Vestnik Moskovskogo gorodskogo pedagogicheskogo universiteta. Seriya Informatiki $i$ informatizatsiya obrazovaniya = Bulletin of Moscow City Teacher Training University. Series Computer Science and Informatization of Education, 2007, no. 2 (9), pp. 156-158. (In Russian).

3. Belozubov A. V., Nikolayev D. G. Sistema distantsionnogo obucheniya Moodle [System of MOOdle distant teaching]. Saint Petersburg National Research University of Information Technologies, Mechanics and Optics (ITMO University) Publ., 2007. 108 p.

4. Bratishchenko V. V., Keshikov K. A. The model with latent parameters for evaluating students'competences on the basis of the current progress marks. Izvestiya Baykal'skogo gosudarstvennogo universiteta = Bulletin of Baikal State University, 2016, vol. 26, no 5, pp. 811-817. DOI: 10.17150/2500-2759.2016.26(5).811-817. (In Russian).

5. Neyman Ju. M., Khlebnikov V. A. Vvedenie $v$ teoriju modelirovanija i parametrizacii pedagogicheskih testov [Introduction to Theory of Modelling and Parametrization of Training Tests]. Moscow, Prometey Publ., 2000. 168 p.

6. Rasch G. Probabilistic Models for Some Intelligence and Attainment Tests. University of Chicago Press, 1980. 199 p.

7. Andrich D. A rating formulation for ordered response categories. Psychometrika, 1978, vol. 43, no. 4, pp. 561-573.

8. Masters G. N. A Rasch model for partial credit scoring. Psychometrika, 1982, vol. 47, no. 2, pp. 149-174.

9. Bratishenko V. V. Parametric model of examination grades. Kachestvo. Innovatsii. Obrazovanie = Quality. Innovations. Education, 2012, no. 3, pp. 32-35. (In Russian).

\section{Информация об авторе}

Братищенко Владимир Владилирович - кандидат физико-математических наук, доцент, заведующий кафедрой информатики и кибернетики, Байкальский государственный университет, 664003, г. Иркутск, ул. Ленина, 11, e-mail: vvb@bgu.ru.

\section{Author}

Vladimir V. Bratishchenko - PhD in Physics and Mathematics, Associate Professor, Chair of Computer Science and Cybernetics, Baikal State University, 11 Lenin St., 664003, Irkutsk, Russian Federation; e-mail: vvb@bgu.ru.

\section{Библиографическое описание статьи}

Братищенко В. В. Информационная образовательная среда Байкальского государственного университета / В. В. Братищенко // Baikal Research Journal. — 2017. — T. 8, № 1. — DOI: $10.17150 / 2411-6262.2017 .8(1) .18$.

\section{Reference to article}

Bratishchenko V. V. Information and educational environment in Baikal State University. Baikal Research Journal, 2017, vol. 8, no. 1. DOI: 10.17150/2411-6262.2017.8(1).18. (In Russian).

\section{Baikal Research Journal}

